\title{
BESII Detector Simulation
}

M. Ablikim ${ }^{\text {a }}$, J.Z. Bai ${ }^{\text {a }, ~ Y . ~ B a n ~}{ }^{\text {b }}$, J.G. Bian ${ }^{\text {a }}$, X. Cai ${ }^{\text {a }}$, J.F. Chang ${ }^{a}$, H.F. Chen ${ }^{c}$, H.S. Chen ${ }^{\mathrm{a}}$, H.X. Chen ${ }^{\mathrm{a}}$, J.C. Chen ${ }^{a}$, Jin Chen ${ }^{a}$, Jun Chen ${ }^{d}$, M.L. Chen ${ }^{a}$, Y.B. Chen ${ }^{a}$, S.P. Chi ${ }^{\text {e, }}$ Y.P. Chu ${ }^{\text {a }}$, X.Z. Cui ${ }^{\text {a }}$, H.L. Dai ${ }^{\text {a }}$, Y.S. Dai ${ }^{\mathrm{f}}$, Z.Y. Deng ${ }^{a}$, L.Y. Dong ${ }^{a}{ }^{a}$, Q.F. Dong ${ }^{\mathrm{g}}$, S.X. Du ${ }^{\mathrm{a}}$, Z.Z. Du ${ }^{\mathrm{a}}$,

J. Fang ${ }^{a}$, S.S. Fang ${ }^{\text {e }}$, C.D. Fu ${ }^{a}$, H.Y. Fu ${ }^{a}$, C.S. Gao ${ }^{\text {a }}$, Y.N. Gao ${ }^{\mathrm{g}}$, M.Y. Gong ${ }^{\mathrm{a}}$, W.X. Gong ${ }^{\mathrm{a}}$, S.D. Gu ${ }^{\mathrm{a}}$, Y.N. Guo ${ }^{\mathrm{a}}$, Y.Q. Guo ${ }^{\text {a }}$, Z.J. Guo ${ }^{\text {h, F.A. Harris }}{ }^{\text {h, K.L. He }}{ }^{\text {a }}$, M. He ${ }^{\mathrm{i}}$, X. He ${ }^{\text {a }}$, Y.K. Heng ${ }^{\text {a }}$, H.M. Hu ${ }^{\text {a }}$, T. Hu ${ }^{\text {a }}$, G.S. Huang ${ }^{\mathrm{a}, 2}$, X.P. Huang ${ }^{\text {a }}$, X.T. Huang ${ }^{\mathrm{i}}$, X.B. Ji ${ }^{\text {a }}$, C.H. Jiang ${ }^{\text {a }}$, X.S. Jiang ${ }^{\text {a }}$, D.P. Jin ${ }^{a}$, S. $\operatorname{Jin}^{\mathrm{a}}$, Y. Jin ${ }^{\mathrm{a}}$, Yi $\mathrm{Jin}^{\mathrm{a}}$, Y.F. Lai ${ }^{\mathrm{a}}$, C.G. Li ${ }^{\mathrm{a}, 3}$, F. Li ${ }^{a}$, G. Li ${ }^{\text {e }}$,H.H. Li ${ }^{\text {a }}$, J. Li ${ }^{\text {a }}$, J.C. Li ${ }^{\text {a }}$, Q.J. Li ${ }^{\text {a }}$, R.Y. Li ${ }^{\text {a }}$, S.M. Li ${ }^{\text {a }}$, W.D. Li ${ }^{\text {a }}$, W.G. Li ${ }^{\text {a }}$, X.L. Li ${ }^{j}$, X.Q. Li ${ }^{k}$, Y.L. Li ${ }^{\ell}$, Y.F. Liang ${ }^{\mathrm{m}}$, H.B. Liao ${ }^{\mathrm{n}}$, C.X. Liu ${ }^{\mathrm{a}}$, F. Liu ${ }^{\mathrm{n}}$, Fang Liu ${ }^{\mathrm{c}}$, H.H. Liu ${ }^{\text {a }}$, H.M. Liu ${ }^{\text {a,* }}$, J. Liu ${ }^{\text {b }, ~ J . P . ~ L i u ~}{ }^{\circ}$, R.G. Liu ${ }^{\text {a }}$, Z.A. $\mathrm{Liu}^{\mathrm{a}}$, Z.X. $\mathrm{Liu}^{\mathrm{a}}$, F. Lu ${ }^{\mathrm{a}}$, G.R. Lu ${ }^{\mathrm{p}}$, H.J. Lu ${ }^{\mathrm{c}}$, J.G. Lu ${ }^{\mathrm{a}}$, C.L. Luo ${ }^{q}$, L.X. $\mathrm{Luo}^{\ell}$, X.L. Luo ${ }^{\mathrm{a}}$, F.C. Ma ${ }^{\mathrm{j}}$, H.L. Ma ${ }^{\mathrm{a}}$, J.M. $\mathrm{Ma}^{\mathrm{a}}$, L.L. $\mathrm{Ma}^{\mathrm{a}}$, Q.M. $\mathrm{Ma}^{\mathrm{a}}$, X.B. Ma ${ }^{\mathrm{p}}$, X.Y. Ma ${ }^{\mathrm{a}}$, Z.P. $\mathrm{Mao}^{\text {a }}$, X.H. Mo ${ }^{\text {a }}$, J. Nie ${ }^{\mathrm{a}}$, Z.D. Nie ${ }^{\mathrm{a}}$, S.L. Olsen ${ }^{\mathrm{h}}$, H.P. Peng ${ }^{\text {c }}$, N.D. Qi ${ }^{\text {a }}$, C.D. Qian ${ }^{\mathrm{r}}$, H. Qin ${ }^{\mathrm{q}}$, J.F. Qiu ${ }^{\mathrm{a}}$, Z.Y. Ren ${ }^{a}$, G. Rong ${ }^{\text {a }}$, L.Y. Shan ${ }^{\text {a }}$, L. Shang ${ }^{\text {a }}$, D.L. Shen ${ }^{\mathrm{a}}$, X.Y. Shen ${ }^{a}$, H.Y. Sheng ${ }^{a}$, F. Shi ${ }^{a}$, X. Shi ${ }^{b, 4}$, H.S. Sun ${ }^{a}$, J.F. Sun ${ }^{a}$, S.S. $\operatorname{Sun}^{a}$, Y.Z. $\operatorname{Sun}^{a}$, Z.J. $\operatorname{Sun}^{a}$, X. Tang ${ }^{a}$, N. Tao ${ }^{c}$, Y.R. Tian ${ }^{\mathrm{g}}$, G.L. Tong ${ }^{\mathrm{a}}$, G.S. Varner ${ }^{\mathrm{h}}$, D.Y. Wang ${ }^{\mathrm{a}}$, J.Z. Wang ${ }^{a}$, K. Wang ${ }^{c}$, L. Wang ${ }^{a}$, L.S. Wang ${ }^{a}$, M. Wang ${ }^{\text {a }}$, P. Wang ${ }^{\text {a }}$, P.L. Wang ${ }^{\text {a }}$, S.Z. Wang ${ }^{\text {a }}$, W.F. Wang ${ }^{\mathrm{a}, 5}$, Y.F. Wang ${ }^{\mathrm{a}}$, Z. Wang ${ }^{\mathrm{a}}$, Z.Y. Wang ${ }^{\mathrm{a}}$, Zhe Wang ${ }^{\mathrm{a}}$, Zheng Wang ${ }^{\text {e, C.L. Wei }}{ }^{a}$, D.H. Wei ${ }^{a}$, N. Wu ${ }^{\text {a }}$, Y.M. Wu ${ }^{\text {a }}$, X.M. Xia $^{a}$, X.X. Xie ${ }^{a}$, B. Xin ${ }^{\mathrm{j}, 6}$, G.F. Xu ${ }^{\text {a }}$, H. Xu ${ }^{\text {a }}$, S.T. Xue ${ }^{\mathrm{a}}$, M.L. Yan ${ }^{\mathrm{c}}$, F. Yang ${ }^{\mathrm{k}}$, H.X. Yang ${ }^{\mathrm{a}}$, J. Yang ${ }^{\mathrm{c}}$, Y.X. Yang ${ }^{\mathrm{s}}$, M. Ye ${ }^{a}$, M.H. Ye ${ }^{e}, Y . X . Y^{c}$, L.H. Yi ${ }^{d}$, Z.Y. Yi ${ }^{a}$, C.S. Yu ${ }^{a}$, G.W. Yu ${ }^{a}$, C.Z. Yuan ${ }^{a}$, J.M. Yuan ${ }^{a}$, Y. Yuan ${ }^{a}$, S.L. Zang ${ }^{a}$, 
Y. Zeng ${ }^{\text {d, }}$ Yu Zeng ${ }^{\text {a }}$, B.X. Zhang ${ }^{\text {a }}$, B.Y. Zhang ${ }^{\text {a }}$, C.C. Zhang ${ }^{\text {a }}$, D.H. Zhang ${ }^{\text {a }}$, H.Y. Zhang ${ }^{\text {a }}$, J. Zhang ${ }^{\text {a }}$, J.W. Zhang ${ }^{\text {a }}$, J.Y. Zhang ${ }^{\text {a }}$, Q.J. Zhang ${ }^{\text {a }}$, S.Q. Zhang ${ }^{\text {a }}$, X.M. Zhang ${ }^{\text {a }}$, X.Y. Zhang ${ }^{\mathrm{i}}$, Y.Y. Zhang ${ }^{\mathrm{a}}$, Yiyun. Zhang ${ }^{\mathrm{m}}$, Z.P. Zhang ${ }^{\mathrm{c}}$, Z.Q. Zhang ${ }^{\text {p }}$, D.X. Zhao ${ }^{\text {a }}$, J.B. Zhao ${ }^{\text {a }}$, J.W. Zhao ${ }^{\text {a }}$, M.G. Zhao ${ }^{\mathrm{k}}$, P.P. Zhao ${ }^{\mathrm{a}}$, W.R. Zhao ${ }^{\mathrm{a}}$, X.J. Zhao ${ }^{\mathrm{a}}$, Y.B. Zhao ${ }^{\text {a }}$, Z.G. Zhao ${ }^{\text {a, }}$, H.Q. Zheng ${ }^{\text {b }}$, J.P. Zheng ${ }^{\text {a }}$, L.S. Zheng ${ }^{\text {a }}$, Z.P. Zheng ${ }^{\text {a }}$, X.C. Zhong ${ }^{\text {a }}$, B.Q. Zhou ${ }^{\text {a }}$, G.M. Zhou ${ }^{a}$, L. Zhou ${ }^{\text {a }}$, N.F. Zhou ${ }^{\text {a }}$, K.J. Zhu ${ }^{\text {a }}$, Q.M. Zhu ${ }^{\text {a }}$, Y.C. Zhu ${ }^{\mathrm{a}}$, Y.S. Zhu ${ }^{\mathrm{a}}$, Yingchun Zhu ${ }^{\mathrm{a}, 8}$, Z.A. Zhu ${ }^{\mathrm{a}}$, B.A. Zhuang ${ }^{\text {a }}$, X.A. Zhuang ${ }^{\text {a }}$, B.S. Zou ${ }^{\text {a }}$

\section{(BES Collaboration)}

anstitute of High Energy Physics, Beijing 100049, People's Republic of China

b Peking University, Beijing 100871, People's Republic of China

${ }^{\mathrm{c}}$ University of Science and Technology of China, Hefei 230026, People's Republic of China

${ }^{\mathrm{d}}$ Hunan University, Changsha 410082, People's Republic of China

${ }^{\mathrm{e} C h i n a}$ Center for Advanced Science and Technology(CCAST), Beijing 100080, People's Republic of China

${ }^{\mathrm{f}}$ Zhejiang University, Hangzhou 310028, People's Republic of China

${ }^{\mathrm{g}}$ Tsinghua University, Beijing 100084, People's Republic of China

${ }^{\mathrm{h}}$ University of Hawaii, Honolulu, Hawaii 96822, USA

iShandong University, Jinan 250100, People's Republic of China

${ }^{\mathrm{j}}$ Liaoning University, Shenyang 110036, People's Republic of China

${ }^{\mathrm{k}}$ Nankai University, Tianjin 300071, People's Republic of China

${ }^{\ell}$ Guangxi University, Nanning 530004, People's Republic of China

${ }^{\mathrm{m}}$ Sichuan University, Chengdu 610064, People's Republic of China

${ }^{\mathrm{n}}$ Huazhong Normal University, Wuhan 430079, People's Republic of China

${ }^{\circ}$ Wuhan University, Wuhan 430072, People's Republic of China

${ }^{\mathrm{p}}$ Henan Normal University, Xinxiang 453002, People's Republic of China

q Nanjing Normal University, Nanjing 210097, People's Republic of China

${ }^{\mathrm{r}}$ Shanghai Jiaotong University, Shanghai 200030, People's Republic of China

${ }^{\mathrm{s}}$ Guangxi Normal University, Guilin 541004, People's Republic of China 


\begin{abstract}
A Monte Carlo program based on Geant3 has been developed for BESII detector simulation. The organization of the program is outlined, and the digitization procedure for simulating the response of various sub-detectors is described. Comparisons with data show that the performance of the program is generally satisfactory.
\end{abstract}

Key words: Monte Carlo simulation, Detector response PACS: 07.05.Tp

\title{
1 Introduction
}

To understand the intrinsic characteristics and performance of a detector, for both detector design and physics analysis in experimental high energy particle physics, reliable Monte Carlo (MC) simulation is essential.

The Beijing Spectrometer (BES $[1,2]$ ) detector started to take data in 1989 at the Beijing Electron Positron Collider (BEPC), which operates in the centerof-mass energy range from 2 to $5 \mathrm{GeV}$. The early simulation program was EGS [3] based and thus did not generate hadronic interactions of the secondary particles in the detector. Some effects, like fake photons produced by hadrons, were not properly described. For the detector response, simple parametric models for sub-detectors were used in the simulation. As the program grew, the hard-coded structure made it difficult to maintain and make improvements.

With the upgrade of the BES detector (BESII) [4] and BEPC in 1996, the development of a new Monte Carlo program, SIMBES, based on the Geant3 package [5], was begun. The decision was motivated by many reasons. First, larger data samples require better detector simulation to reduce systematic uncertainties. Second, some sub-systems of BESII were partly changed and some totally new, and it was difficult to make the required software changes within the old program structure. Meanwhile, the Geant framework had proven to be

\footnotetext{
* Corresponding author

Email address: liuhm@ihep.ac.cn (H.M. Liu).

1 Current address: Iowa State University, Ames, Iowa 50011-3160, USA.

2 Current address: Purdue University, West Lafayette, Indiana 47907, USA.

3 Current address: 12175 Eastbourne Road, San Diego, California 92128, USA.

4 Current address: Cornell University, Ithaca, New York 14853, USA.

5 Current address: Laboratoire de l'Accélératear Linéaire, F-91898 Orsay, France.

6 Current address: Purdue University, West Lafayette, Indiana 47907, USA.

7 Current address: University of Michigan, Ann Arbor, Michigan 48109, USA.

8 Current address: DESY, D-22607, Hamburg, Germany.
} 
a powerful tool for detector description and particle tracking in high energy physics.

Geant3.21, the last version of Geant3, is used in SIMBES. Much effort is made to model the signal generation, since the digitization procedure is highly detector dependent. BESII data is used for tuning parameters to describe the detector response. Many physics channels are compared with data to check the simulation, and the agreement is reasonably good. In this paper, the general organization and features of SIMBES are described in Section 2. Detailed simulations of detector response, as well as comparisons with data, are presented in Section 3.

\section{The BESII Simulation Program - SIMBES}

\subsection{The BESII Detector}

BESII is a conventional solenoidal magnetic detector that is described in detail in Ref. [4]. A 12-layer vertex chamber (VC) surrounding the beam pipe provides trigger and coordinate information. A 40-layer main drift chamber (MDC), located radially outside the VC, provides trajectory and energy loss $(\mathrm{d} E / \mathrm{d} x)$ information for charged tracks over $85 \%$ of the total solid angle. The momentum resolution is $\sigma_{p} / p=0.017 \sqrt{1+p^{2}}(p$ in $\mathrm{GeV} / \mathrm{c})$, and the $\mathrm{d} E / \mathrm{d} x$ resolution for hadron tracks is $\sim 8 \%$. An array of 48 scintillation counters surrounding the MDC measures the time-of-flight (TOF) of charged tracks with a resolution of $\sim 200$ ps for hadrons. Radially outside the TOF system is a 12 radiation length, lead-gas barrel shower counter (SC). This measures the energies of electrons and photons over $80 \%$ of the total solid angle with an energy resolution of $\sigma_{E} / E=22 \% / \sqrt{E}$ ( $E$ in $\mathrm{GeV}$ ). Outside of the solenoidal coil, which provides a 0.4 Tesla magnetic field over the tracking volume, is an iron flux return that is instrumented with three double layers of counters which identify muons with momentum greater than $0.5 \mathrm{GeV} / \mathrm{c}$.

The geometrical and material descriptions of the detector are given in Section 3. The experimentally measured field map is used in the simulation.

\subsection{Program Structure}

Built into the design of Geant is the separation of geometry, tracking, and digitization. SIMBES respects this feature as illustrated in Fig. 1. In the initialization stage, three kinds of data files are loaded: the material and geometry 


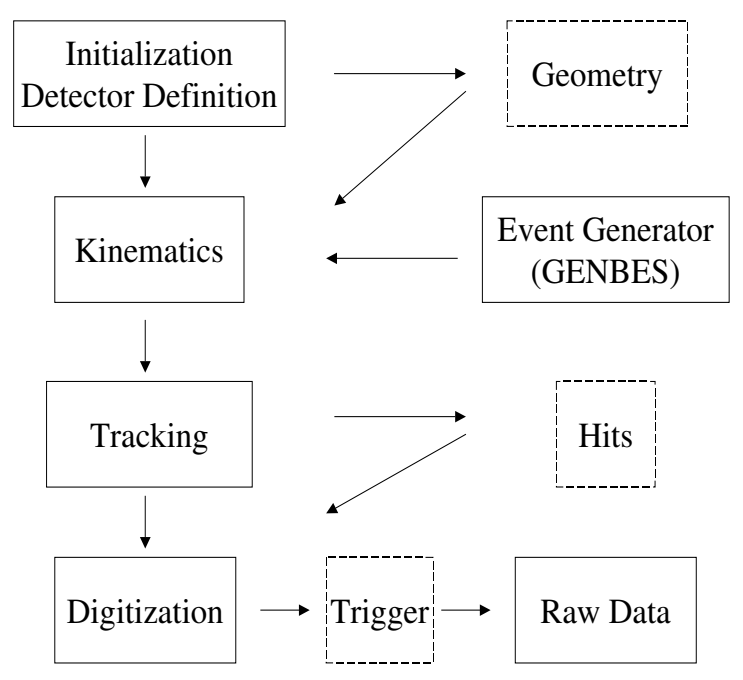

Fig. 1. Schematic structure of SIMBES, dashed boxes mean optional.

configuration data used to define the detector, the digitization constants to describe the detector response, and the realization constants (such as dead channels and wire efficiency) to simulate the real detector performance. Input values of these parameters can be easily changed by experts or users using control cards without relinking the program.

The BESII event generator package (GENBES) is a stand-alone program that can be executed from within SIMBES to provide kinematic quantities for primary events. The generators for radiative QED processes [6], selected decays of $J / \psi, \psi(2 S)$, and charmed meson pair production [7] were taken from other experiments. The BES developed generators, LUND-CRM for inclusive decays of $J / \psi$ and $\psi(2 S)$ [8] and PPGEN for some exclusive decays of the $\psi(2 S)$, as well as some standard generators like JETSET, are also included in GENBES.

At the end of each stage of processing (see Fig. 1), the intermediate output (the geometry or hit information in the Geant data structure) can be saved to a disk file, and used as input for later SIMBES execution. SIMBES code structure is hierarchical and modularized. Therefore, each sub-detector stands alone and can be turned on or off by the user. The BESII trigger logic is also implemented in a single module; the user has an option to switch it on or off when the digitization stage is finished. SIMBES is usually run in batch mode to generate raw data and "MC-truth" data. The interactive mode is used for graphic event display. 


\subsection{Tracking and Hits}

The tracking of particles through the BESII detector is essentially done according to the Geant standard. Tracking cutoffs and parameters are carefully tuned for each sub-detector to provide a compromise between simulation precision and computing speed. To correctly simulate electromagnetic shower development in the shower counter, the kinetic energy cutoffs of electrons and photons have been set to Geant's limit (10 KeV).

When a charged particle traverses a sensitive volume (a cell or readout channel), the hit information, such as the particle position, energy deposit, etc., are saved for the digitization procedure which is discussed in the next section.

\subsection{Hadronic Interaction}

For hadronic interactions, several models have been tried and compared with BESII data; each has advantages for specific channels. Fluka [9] and Gcalor [10] are the two hadronic models that users can choose in SIMBES.

Extra photons produced by hadronic interactions in the shower counter are studied by a very clean channel $\psi(2 S) \rightarrow \pi^{+} \pi^{-} J / \psi \rightarrow \pi^{+} \pi^{-} \mu^{+} \mu^{-}$(only 4 charged tracks in the final state). The neutral tracks observed come mainly from pion hadronic interactions in the SC material. Fig. 2 shows comparisons
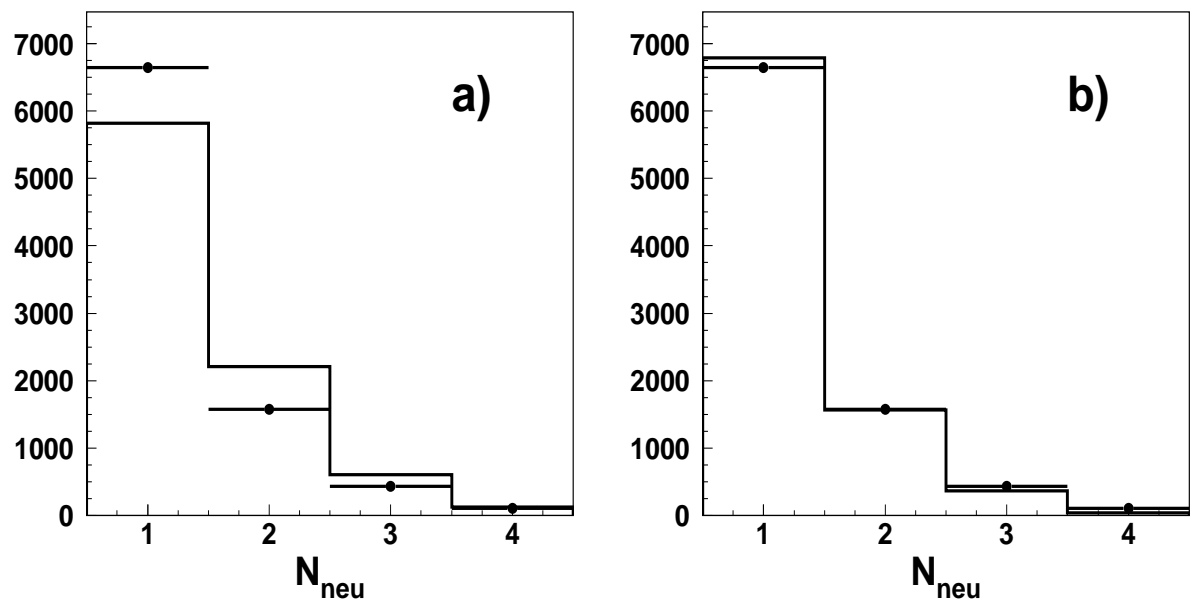

Fig. 2. Number of neutral tracks in the shower counter for $\psi(2 S) \rightarrow \pi^{+} \pi^{-} J / \psi \rightarrow \pi^{+} \pi^{-} \mu^{+} \mu^{-}$decays. Histograms are for MC data with (a) Fluka or (b) Gcalor, and points with error bars are for data.

between data and MC data for different hadronic packages. Gcalor reproduces the number of neutral tracks in the SC better than Fluka. 
In Fluka, inelastic cross sections for anti-nucleon-nucleus annihilation are set to zero if the anti-nucleon $(\bar{n}$ or $\bar{p}$ ) kinetic energy is below $50 \mathrm{MeV}$ (corresponding to a momentum of $310 \mathrm{MeV} / \mathrm{c}$ ); this is, of course, not satisfactory for the BEPC energy domain. Therefore, the parametrization given in [11] is used instead to approximate the cross-sections for anti-nucleons at low momentum. For other particles and for elastic cross-sections, standard Fluka is used. Fig. 3 shows the energy deposition in the $\mathrm{SC}$ for low momentum (less than 0.8
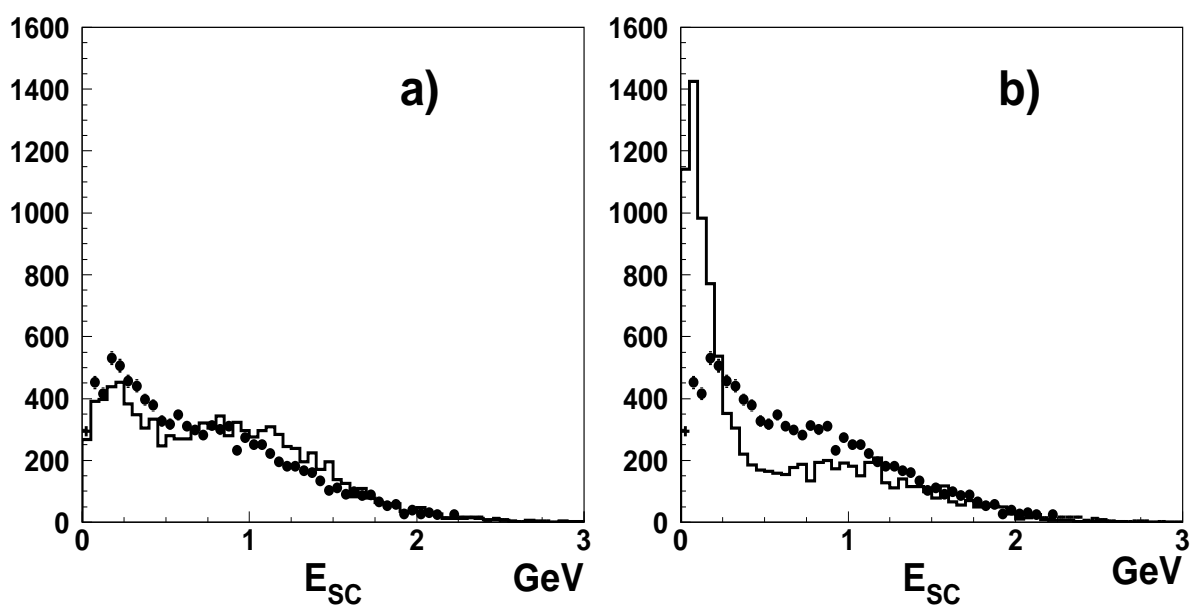

Fig. 3. Energy deposited by $\bar{p}$ in the shower counter for $J / \psi \rightarrow \pi^{+} \pi^{-} p \bar{p}$ decays. Histograms are for MC data with (a) Fluka or (b) Gcalor, and points with error bars are for data.

$\mathrm{GeV} / \mathrm{c}$ ) anti-protons for $J / \psi \rightarrow \pi^{+} \pi^{-} p \bar{p}$ decays. Fluka's simulation is better than Gcalor's.

\section{Simulation of Detector Response}

\subsection{Vertex of event and Vertex Chamber}

The location of the primary event vertex is determined by the interaction point (IP) of the beam bunches, which varies with each beam collision. The IP location and beam size distributions are obtained from experimental data for different running periods, and they are used to sample the primary vertex in $\mathrm{MC}$ event generation. A $T_{0}$ fluctuation caused by the extended beam bunch size is taken into account in the TOF simulation (see Section 3.3).

The vertex chamber (VC), which is built around the beryllium beam-pipe, is composed of 12 layers of straw-tubes. Its hit (time) information is used for the trigger. When a charged particle traverses a straw-tube, the minimum distance between the track and the wire is taken as the drift distance and 
smeared according to the wire space resolution. The drift time of the ionization electrons is obtained by dividing the drift distance by an effective drift velocity. The wire efficiency and the wire sag due to gravity are also taken into consideration in this calculation. The VC simulation is checked using the decay $J / \psi \rightarrow K_{S} K_{L}$ [12], which may lose events in the VC trigger because of the long decay length of the $K_{S}$ particles. The agreement between data and $\mathrm{MC}$ simulation is good when the VC trigger is applied (Fig. 4).
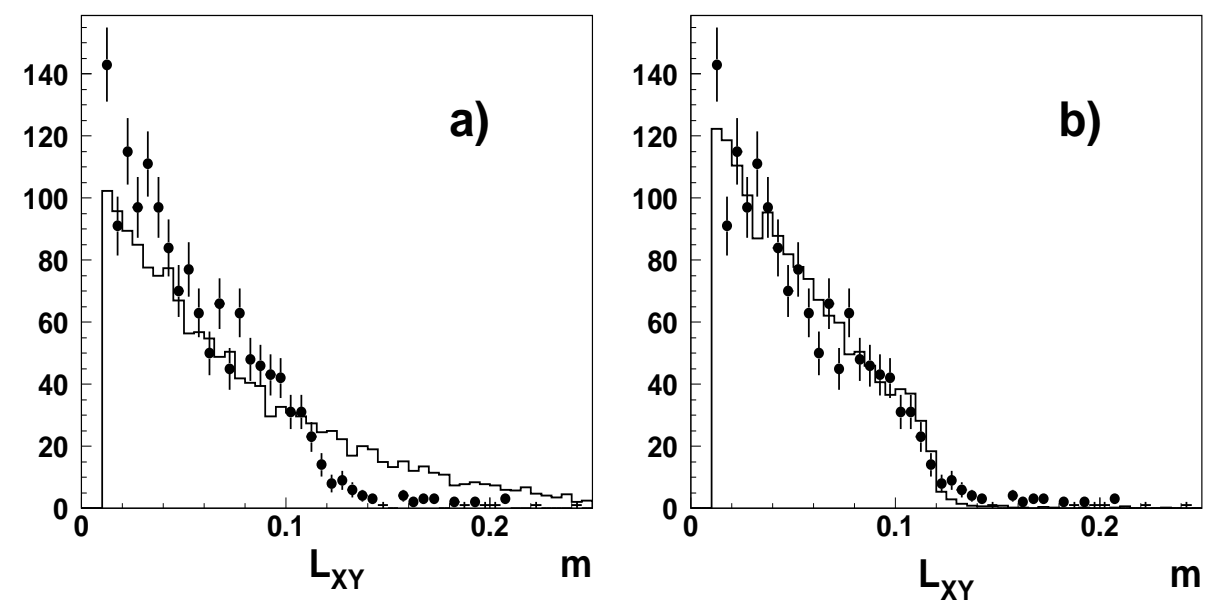

Fig. 4. Comparison of the $K_{S}^{0}$ (from $J / \psi \rightarrow K_{S} K_{L}$ decays) decay length distributions between data and $\mathrm{MC}$ data. Histograms are for $\mathrm{MC}$, and points with errors for data (a) without the trigger and (b) with the trigger.

\subsection{Main Drift Chamber}

The main drift chamber, which is used to determine trajectories and measure energy losses of charged particles, is the main tracking detector of BESII. It is cylindrical with 10 super layers with 4 sense wires (axial or stereo) in each superlayer. The axial layer is described by Geant's TUBE volume. For stereo layers, the shape of the hyperbolic tube (HYPE) in Geant cannot reproduce the geometry. Instead, the subtraction technique is used on two HYPEs for the stereo-layer description. In the simulation, the wires (sense, field and guard) are not put into SIMBES individually, but an equivalent material inside the MDC, taken as a mixture of gas and wires, is used.

The energy loss calculated by Geant for a thin layer is not used in simulating the energy loss by charged tracks in the MDC. Instead, the energy loss (Q) at a sense wire is simulated according to the distribution obtained from real data, smeared with an experimentally determined Landau distribution. Fig. 5 shows $\mathrm{d} E / \mathrm{d} x$ distributions for different particles, where $\mathrm{d} E / \mathrm{d} x$ is the truncated mean of the energy loss for each track. 

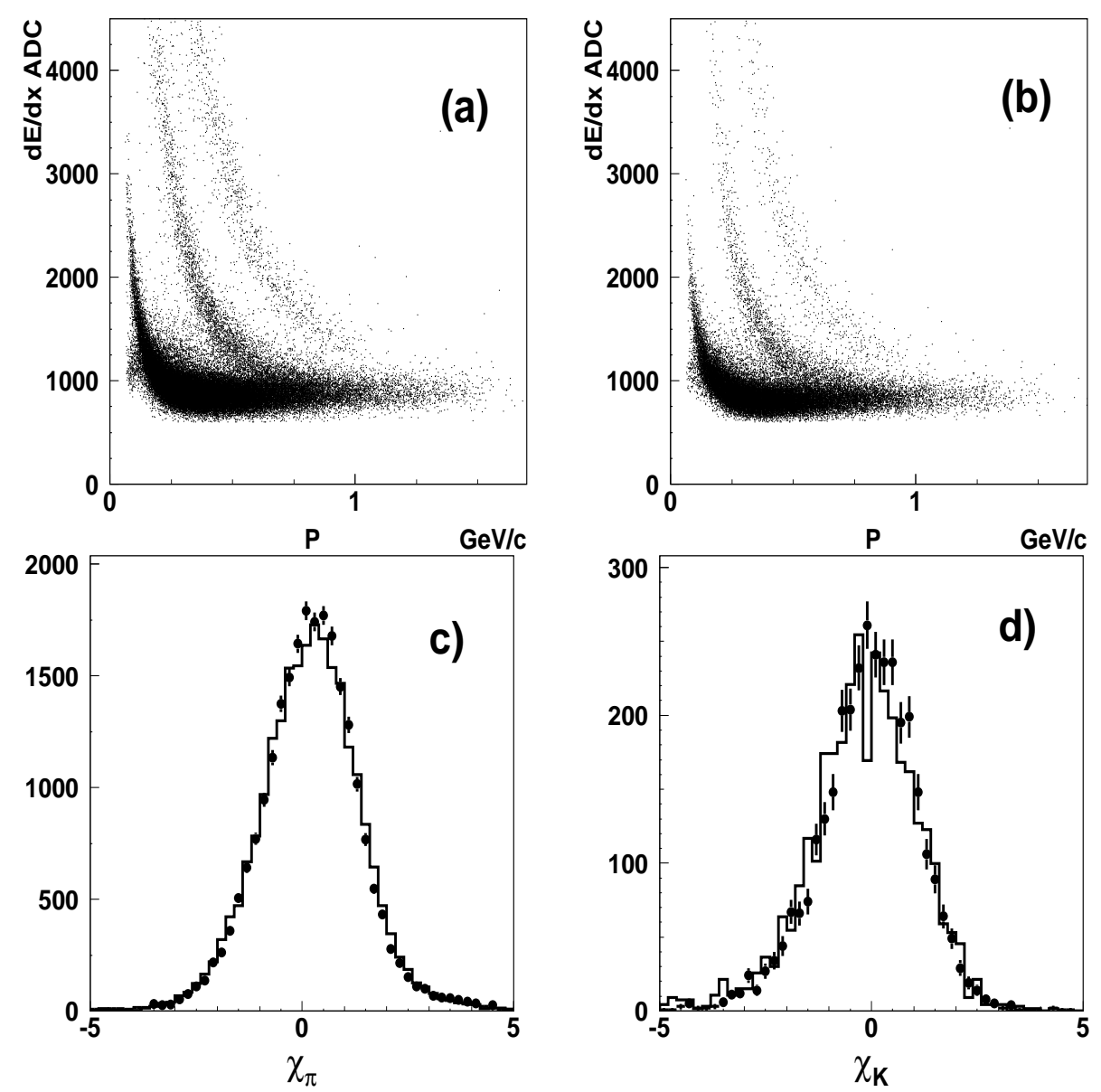

Fig. 5. Top: $\mathrm{d} E / \mathrm{d} x$ (pulse-height) versus momentum for (a) $\mathrm{MC}$ and (b) data. Bottom: The $\left.\chi_{i}\left((\mathrm{~d} E / \mathrm{d} x)_{\text {measured }}-(\mathrm{d} E / \mathrm{d} x)_{\text {expected }}\right) / \sigma\right)$ distributions for $(\mathrm{c}) \pi$ (d) $K$ particles from inclusive $J / \psi$ decays. Histograms are for MC data, and points with error bars are for data.

Using wire hit information, the minimum distance between a track and each wire is calculated including the effects of the Lorentz angle $\left(\alpha_{L}=26^{\circ}\right)$ and gravitational sagging of the wires. The drift distance of the ionization is taken as the minimum distance and is smeared by the wire space resolution $\left(\sigma_{w}\right)$. It is observed from data that the residual of the drift distance is dependent on $\mathrm{Q}$, and even for a given Q, it cannot be simply fitted with a single Gaussian (see Fig. 6). Therefore, the drift distance is smeared according to a combined distribution of two Gaussians (fit from data) with the amount of smearing determined by the $\mathrm{Q}$ value and tracking layer. The wire efficiency is included in the $\sigma_{w}$ simulation. When there are multiple hits in a cell, the time signal is taken as the minimum drift time, while the pulse height is determined from the sum of energy losses.

Fig. 7 shows momentum distributions (after track reconstruction) for data and MC data. The comparison of momentum resolutions for data and Monte Carlo is shown in Table 1. The pion tracking efficiency (see Fig. 8) for simulated 

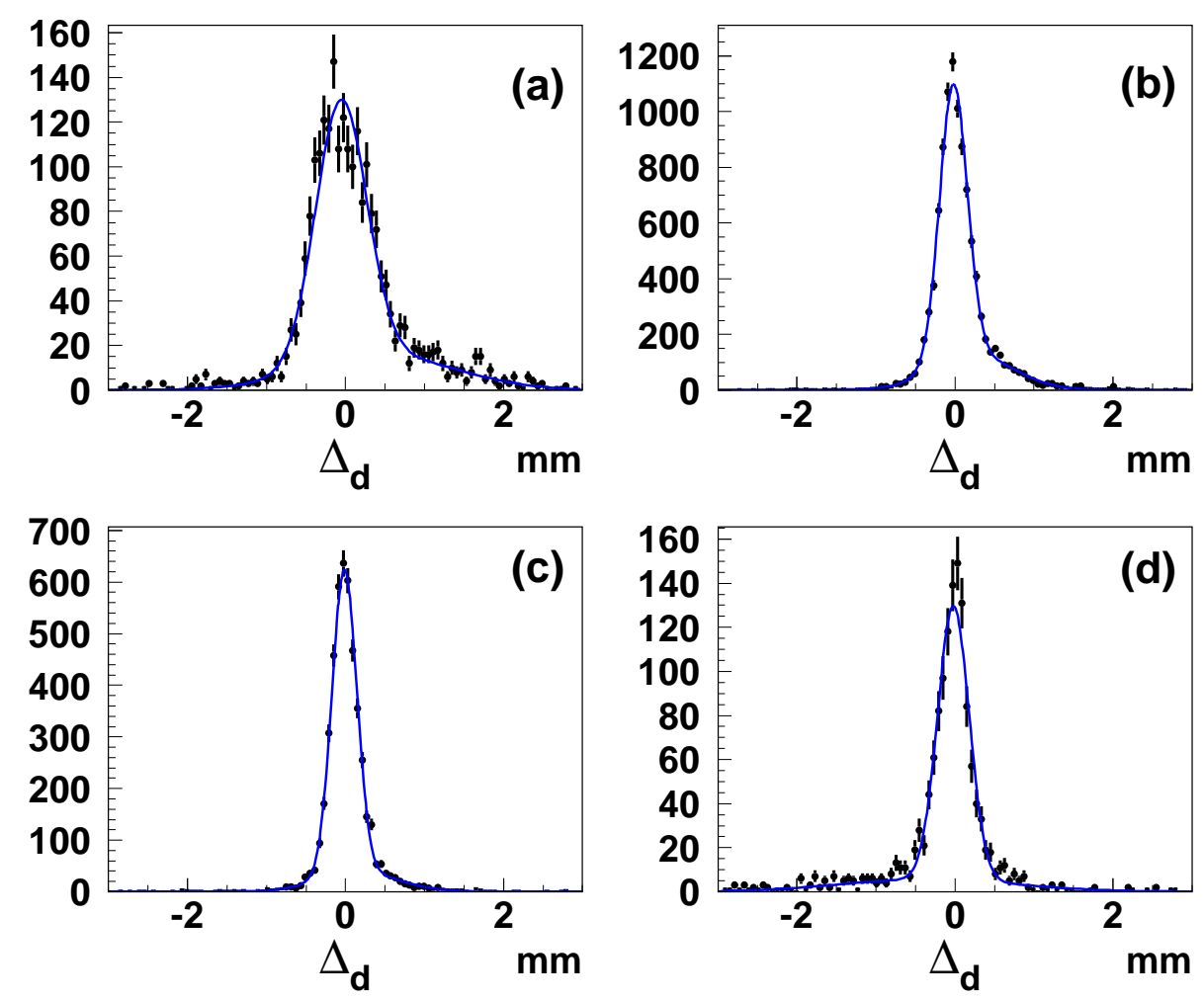

Fig. 6. Distributions of drift distance residuals $\Delta_{d}$ for different pulse heights, Q, corresponding to ADC counts of (a) 100-500, (b) 900-1100, (c) 1900-2100, and (d) 3700-4000. Points with error bars are from Bhabha events at the $J / \psi$, and curves are fits with combined distributions of two Gaussians.
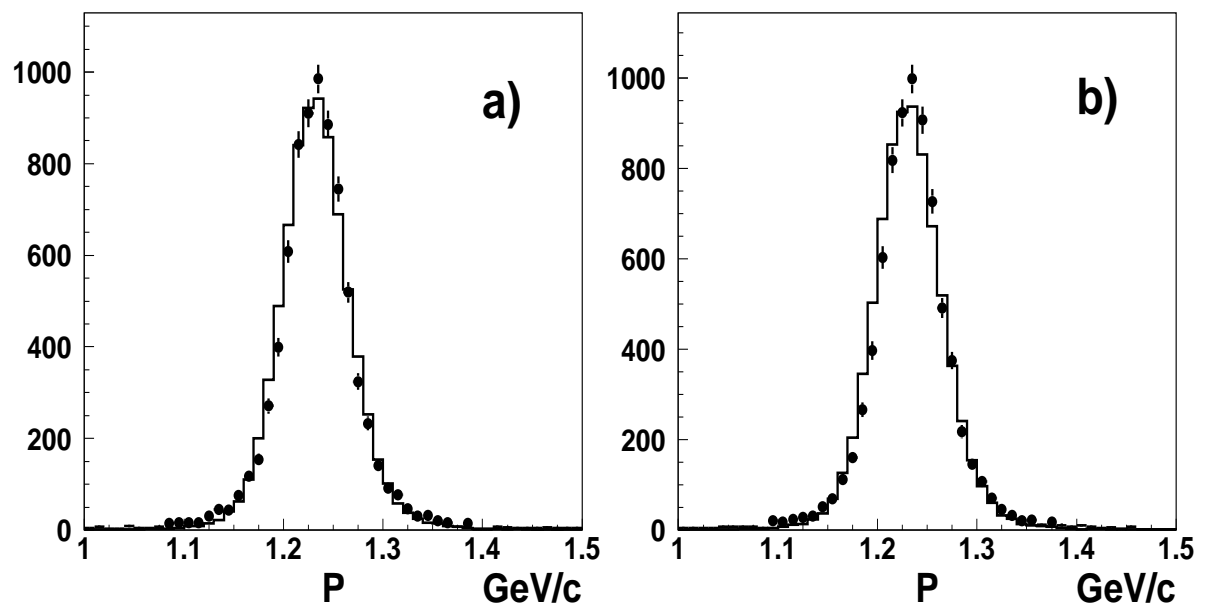

Fig. 7. Momentum distributions for (a) protons and (b) anti-protons from $J / \psi \rightarrow p \bar{p}$ decay. Histograms are for MC, and points with error bars are for data.

data is also found to be consistent with data. In general, an error of $2 \%$ per track is taken as the systematic error from the tracking efficiency. Alternative algorithms for the MDC wire resolution simulation have also been tested, and their performances are slightly worse; however, they can be used to determine the systematic uncertainties from the MDC wire resolution simulation. 
Table 1

Comparison of momentum resolutions for data and Monte Carlo data.

\begin{tabular}{c|c|c}
\hline \hline Channel & $\sigma_{M C}(\mathrm{MeV} / \mathrm{c})$ & $\sigma_{D T}(\mathrm{MeV} / \mathrm{c})$ \\
\hline$e^{+} e^{-} \rightarrow \mu^{+} \mu^{-}$at $J / \psi$ & $47.01 \pm 0.06$ & $46.70 \pm 0.08$ \\
\hline$J / \psi \rightarrow p \bar{p}$ & $33.22 \pm 0.28$ & $34.18 \pm 0.12$ \\
\hline \hline
\end{tabular}

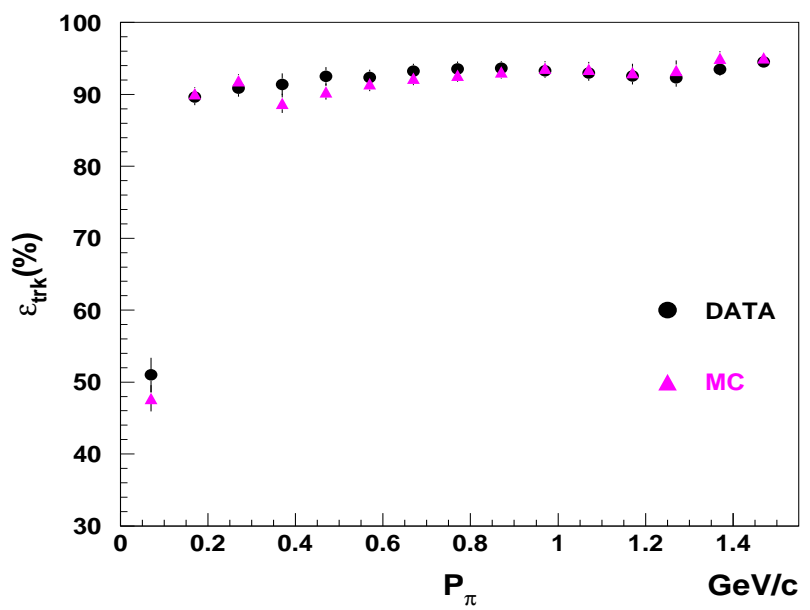

Fig. 8. $\pi$ tracking efficiency in the MDC versus momentum. The first three points are from $J / \psi \rightarrow \Lambda \bar{\Lambda} \rightarrow \pi^{+} \pi^{-} p \bar{p}$, and the others are from $J / \psi \rightarrow \pi^{+} \pi^{-} \pi^{0}$.

\subsection{Time of Flight System}

The TOF system provides precise time measurements for particle identification and the trigger. It consists of two parts, the barrel (BTOF) and the end-caps (ETOF). The BTOF, located just outside of the MDC, employs 48 scintillation counters with photo-multiplier tubes (PMT) at each end. Each ETOF contains 24 scintillators with PMTs at one end. In SIMBES, the TOF system is described simply by trapezoidal scintillator bars with small gaps between adjacent ones.

After the track passes through the scintillator, the TOF hit information contains the particle's position, $Z$, at the entry point, its energy deposition, $E_{\text {loss }}$, in the scintillator, and the time of flight, $T_{f}$, from the IP. $E_{\text {loss }}$ is determined using energy loss with $\delta$-ray generation and applying Birk's law for saturation, while for $T_{f}$, the time spread due to the sizable beam bunch is taken into account. A parameterized model is used to simulate the ADC and TDC outputs; it assumes the light produced at the entry point propagates with attenuation to the two ends. The amplitude and arrival time are obtained according to

$$
Q=f \times E_{\text {loss }} \times \exp \left(Z / L_{\text {atte }}\right), T=T_{f}+Z / v_{c}+T_{c}(Q, Z),
$$

where $\mathrm{f}$ is a conversion constant, $L_{\text {atte }}$ the attenuation length (about $\left.3 \mathrm{~m}\right), v_{c}$ 
the effective light velocity in scintillator, the last term in $T$ is a correction for $Q$ and $Z$ which is obtained from the calibration. The time walk effect is included in $T_{c}$.

ADC and TDC counts are generated by smearing $Q$ and $T$ according to their resolutions, and a $Q$ threshold is applied to the signal. The resolutions for each counter are obtained using Bhabha events; in the smearing, the time resolution dependence with amplitude and hit position is taken into account. For multiple hits in a single counter, taking the time walk effect as a good approximation, only the hits with $Q$ over threshold contribute to the TDC count, while all pulse heights are summed to give the ADC count.

Fig. 9 shows the $\Delta T$ distributions for Bhabha and dimuon events, and the
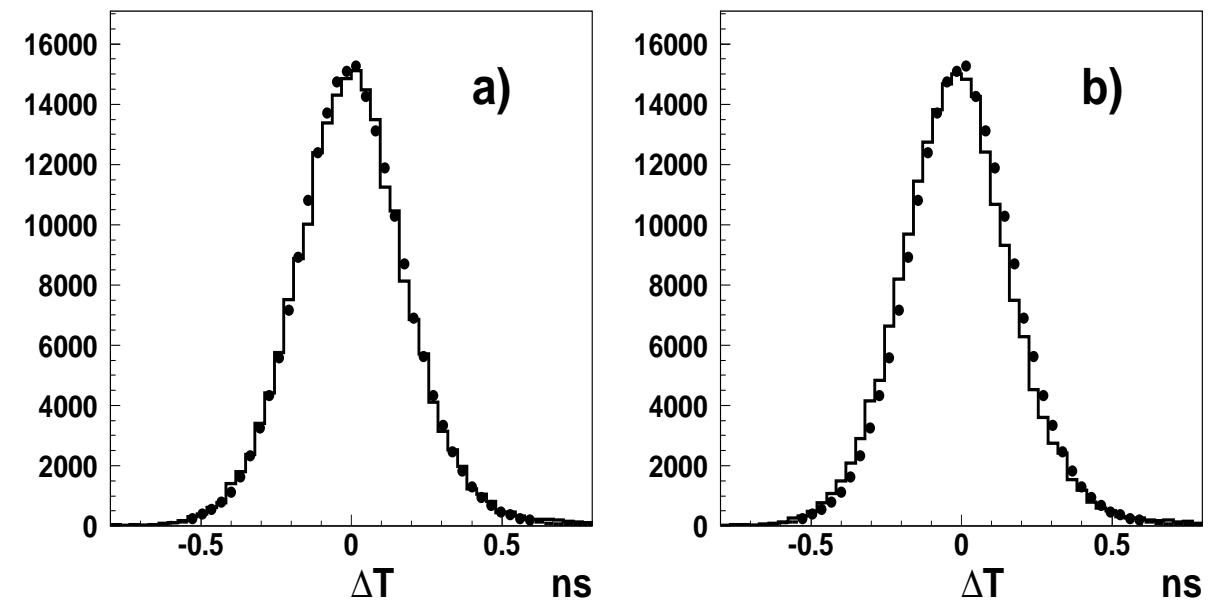

Fig. 9. $\Delta T$ ( $\left.T_{\text {measured }}-T_{\text {expected }}\right)$ distributions for (a) Bhabha and (b) dimuon events at the $J / \psi$ energy. Histograms are for MC data, and points with error bars are for data.

pion particle identification (PID) efficiency for the BTOF as a function of momentum is shown in Fig. 10. The simulation is in a good agreement with data, and an uncertainty of $1 \%$ per track is usually taken as the systematic error for PID with the TOF.

\subsection{Shower Counter}

The shower counter consists of two parts, the barrel (BSC) and the endcaps (ESC). In this section, BSC is taken as an example to demonstrate the simulation procedure. The cylindrical BSC consists of 24 layers of gas tubes interleaved with 23 layers of lead absorbers. There are 560 cells (tubes made of Aluminum) in each gas layer. In order to reduce the number of electronic readout channels, layers with the same $\phi$ angle are grouped into six readout layers in the $r$ direction. To save computation time, the absorber layer is 


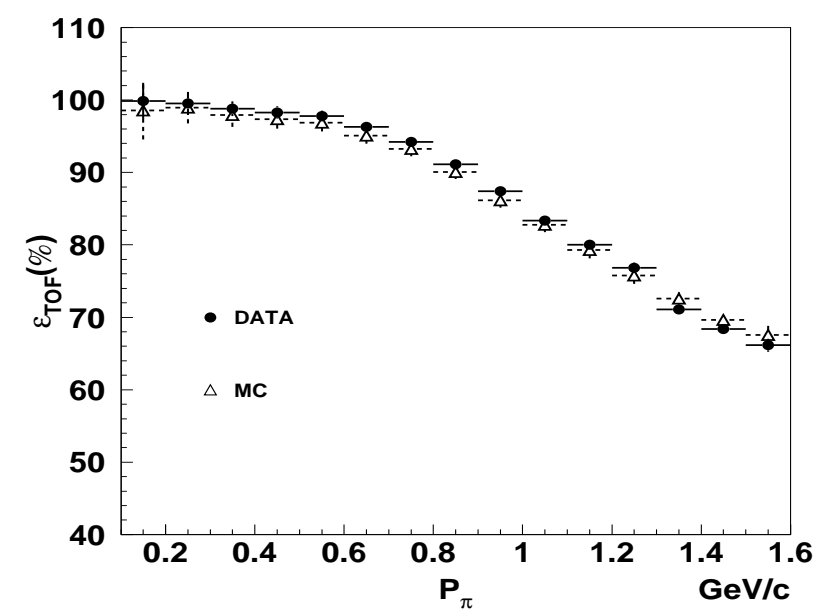

Fig. 10. Pion identification efficiency as a function of momentum for the TOF from $\left.J / \psi \rightarrow \pi^{+} \pi^{-} \pi^{0}\right)$.

modeled using a mixture of $\mathrm{Al}-\mathrm{Pb}-\mathrm{Al}$ sandwiches as its material, while the sensitive layer (cell) is filled with gas working in self-quenching streamer (SQS) mode. The support ribs and some insensitive regions around them are properly described in SIMBES.

When a charged particle hits a gas tube, the energy deposited (total charge) is sampled according to the SQS spectrum which can be parameterized by a Landau distribution. Corrections are made to this charge for the angle dependence of the track and for different layers. The pulse heights at the two ends of the tube are derived by charge division, and the final ADC outputs are obtained by smearing the pulse heights with a Gaussian which accounts for the contribution from the electronics. In the case of multiple hits in a tube, if two hits are close enough, they are merged as one hit.

The simulated hit profiles (number of hits in different layers) of an electromagnetic shower are in close agreement with data. Fig. 11 shows the energy deposition (using Gcalor) in the BSC for different particles after the energy calibration with Bhabha events. Fig. 12 [13] shows the ratio of photon detection efficiency in the BSC for data and MC data. The comparison indicates reasonable agreement, and $2 \%$ per photon is taken as the systematic error for the photon detection efficiency.

\subsection{Muon Counter}

The muon identifier is the outermost component of BES and is composed of three layers of iron absorber and three layers of proportional chambers. There are 189 chambers distributed in an octagonal structure, each chamber consists of 8 proportional gas tubes which are arranged into two sub-layers. 

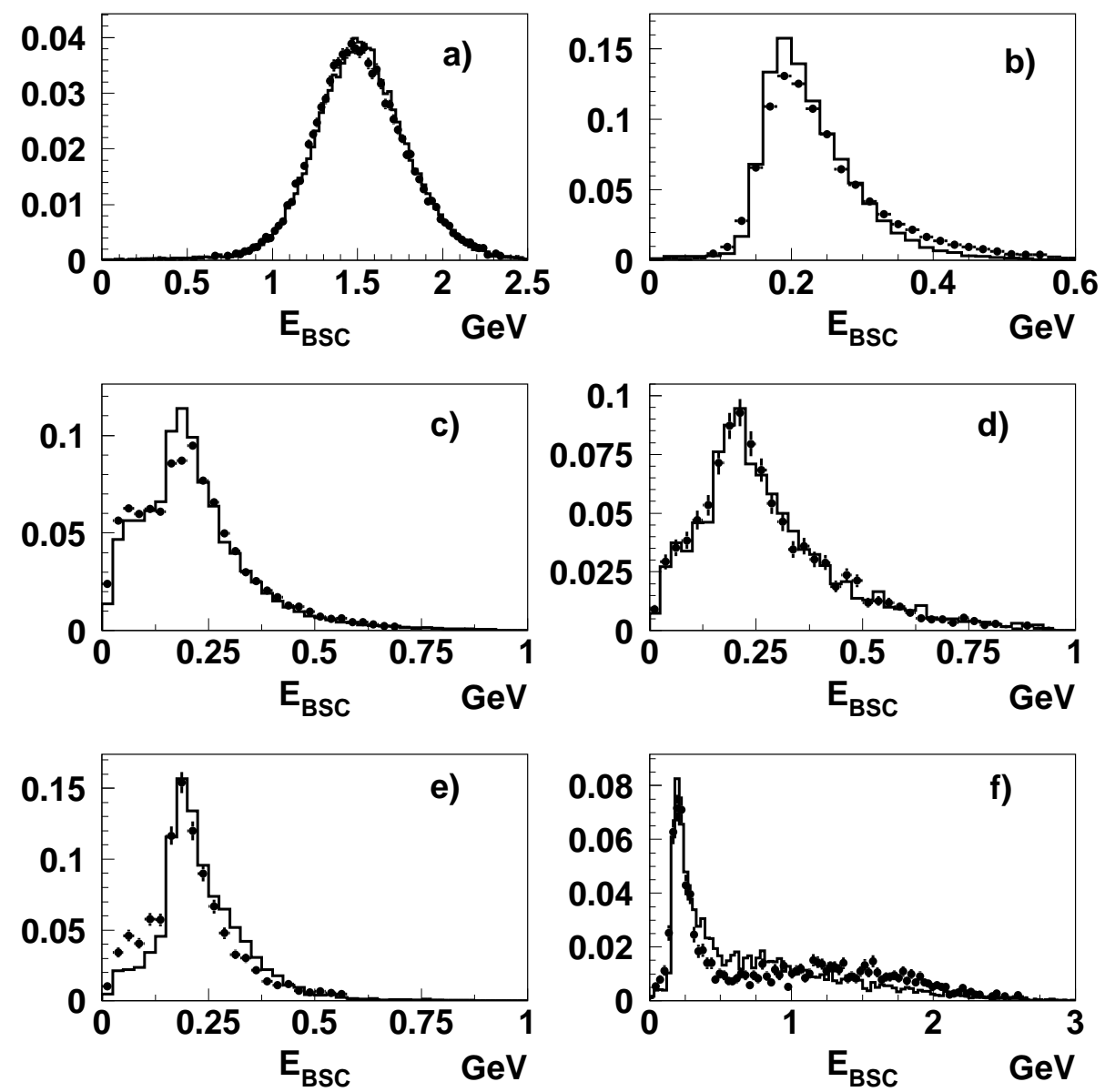

Fig. 11. Particle energy deposited in the BSC, (a) and (b) for $e$ and $\mu$ from Bhabha and dimuon events at the $J / \psi,(\mathrm{c})$ and (d) for $\pi$ and $K$ from inclusive $J / \psi$ decays, and (e) and (f) for $p$ and $\bar{p}$ from $J / \psi \rightarrow p \bar{p}$. Histograms are for MC data, and points with error bars are for data.

The complex geometrical structure and magnetic field [14] of the muon system are well described in SIMBES.

In the simulation, the hit position $(\mathrm{Z})$ along the tube is smeared by the resolution (obtained from data), the ADC signals at the two ends are obtained from charge division of the energy deposit from Geant, and the wire efficiency from data is also included. Fig. 13 shows the comparison of the hit positions in the muon chamber for data and MC data. The agreement is satisfactory.

\section{Summary}

A Monte Carlo program SIMBES has been developed for BESII simulation. Special efforts are made to accurately simulate the response of the detector. For each sub-detector, some key physics quantities are compared with BE- 


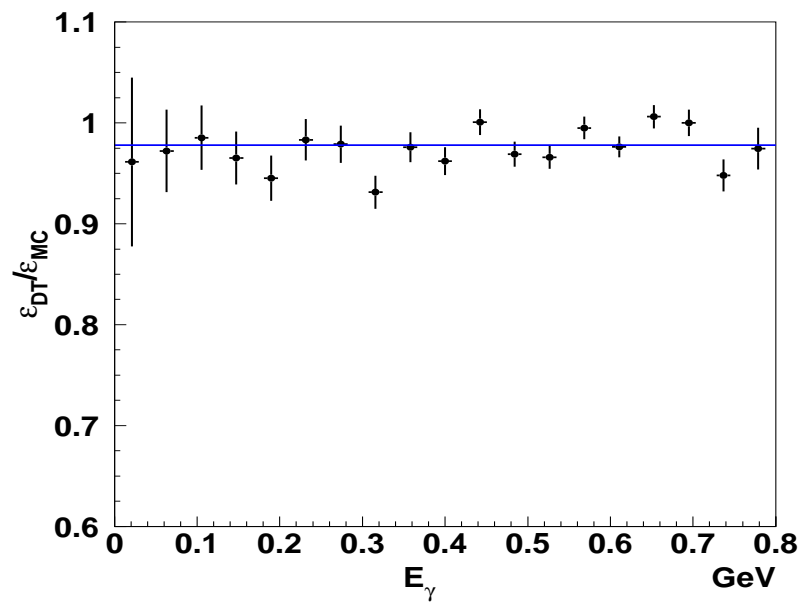

Fig. 12. The ratio of photon detection efficiency between data and $\mathrm{MC}\left(\epsilon_{D T} / \epsilon_{M C}\right)$ versus photon energy. $\epsilon_{D T}$ and $\epsilon_{M C}$ are calculated using $J / \psi \rightarrow \pi^{+} \pi^{-} \pi^{0}$ decays. The solid line is a fit to the points.
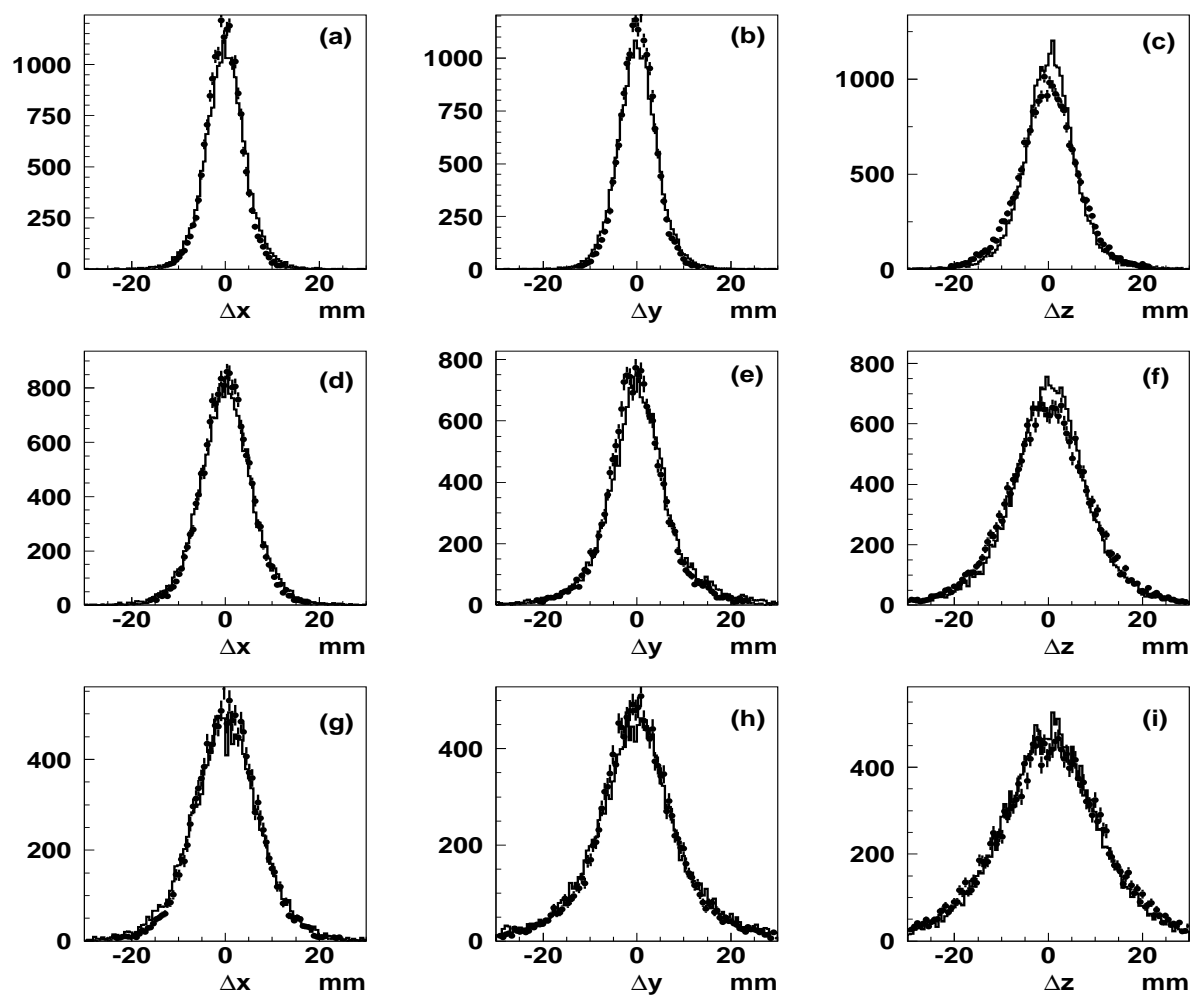

Fig. 13. Hit position residuals for three Muon layers; the top, middle, and bottom plots correspond to layers 1,2 , and 3, respectively. Muons are from $e^{+} e^{-} \rightarrow \mu^{+} \mu^{-}$ at $J / \psi$ events. Histograms are for MC data, and points with error bars are for data.

SII data to test the simulation quality. It is shown from many checks that the overall performance of the current SIMBES is satisfactory and that the results from simulation are generally consistent with data. On the other hand, there is still room for further improvement. For example, the digitization of MDC should be regarded as an 'effective' simulation where many physics mecha- 
nisms are hidden, and noise in the MDC is not considered at present.

The new Monte Carlo package (SIMBES) has significant impact on physics results. As an example, the branching ratio of $J / \psi \rightarrow \pi^{+} \pi^{-} \pi^{0}$ measured by BES is $(2.10 \pm 0.12) \%$ [15], which is significantly higher than the PDG value of $(1.50 \pm 0.20) \%$ [16]. In this measurement, it is found that the efficiency using a two-Gaussian resolution function in the MDC wire simulation is about $20 \%$ lower than that using a single Gaussian function, and that the efficiency including hadronic interactions is about $10 \%$ lower than without them.

\section{Acknowledgements}

We would like to thank Dr. S. Banerjee for helpful discussions and suggestions. We also acknowledge the contribution of many other past members of the BES Collaboration. This work is supported in part by the National Natural Science Foundation of China under contracts Nos. 10491300, 10225524, 10225525, the Chinese Academy of Sciences under contract No. KJ 95T-03, the 100 Talents Program of CAS under Contract Nos. U-11, U-24, U-25, and the Knowledge Innovation Project of CAS under Contract Nos. U-602, U-34 (IHEP); by the National Natural Science Foundation of China under Contract No. 10175060 (USTC), and No. 10225522 (Tsinghua University); and by the Department of Energy under Contract No. DE-FG02-04ER41291 (U Hawaii).

\section{References}

[1] J.Z. Bai, et al. (BES Collab.), Nucl. Instr. and Meth. A 344 (1994) 319.

[2] Ding Hui-Liang, et al. (BES Collab.), HEP \& NP 16 (1992) 769.

[3] W.R. Nelson, H. Hirayama and D.W.O. Rogers, The EGS4 Code System, SLAC-265, 1992.

[4] J.Z. Bai, et al. (BES Collab.), Nucl. Instr. and Meth. A 458 (2001) 627.

[5] CERN Program Library Long Writeup W5013, CERN, 1993.

[6] The generators, written by F.A. Berends et al., include the processes of radiative Bhabha, radiative muon pair and radiative two photons.

[7] The generators were originally developed by the MARKIII Collaboration.

[8] J.C. Chen, et al., Phys. Rev. D 62 (2000) 034003.

[9] A. Ferrari, P.R. Sala, G. Guaraldi and F. Padoani, Nucl. Instr. Meth. B 71 (1992) 412. 
[10] C. Zeitnitz and T.A. Gabriel, Nucl. Instr. and Meth. A 349 (1994) 106.

[11] M. Astrua, et al. Nucl. Phys. A 697 (2002) 209.

[12] X. Shi, et al., HEP \& NP 28 (2004) 982.

[13] S.M. Li, et al., HEP \& NP 28 (2004) 859.

[14] Z. Wang, et al., HEP \& NP 27 (2003) 716.

[15] J.Z. Bai, et al. (BES Collab.), Phys. Rev. D 70 (2004) 012005.

[16] Particle Data Group, S. Eidelman, et al., Phys. Lett. B 592 (2004) 60. 